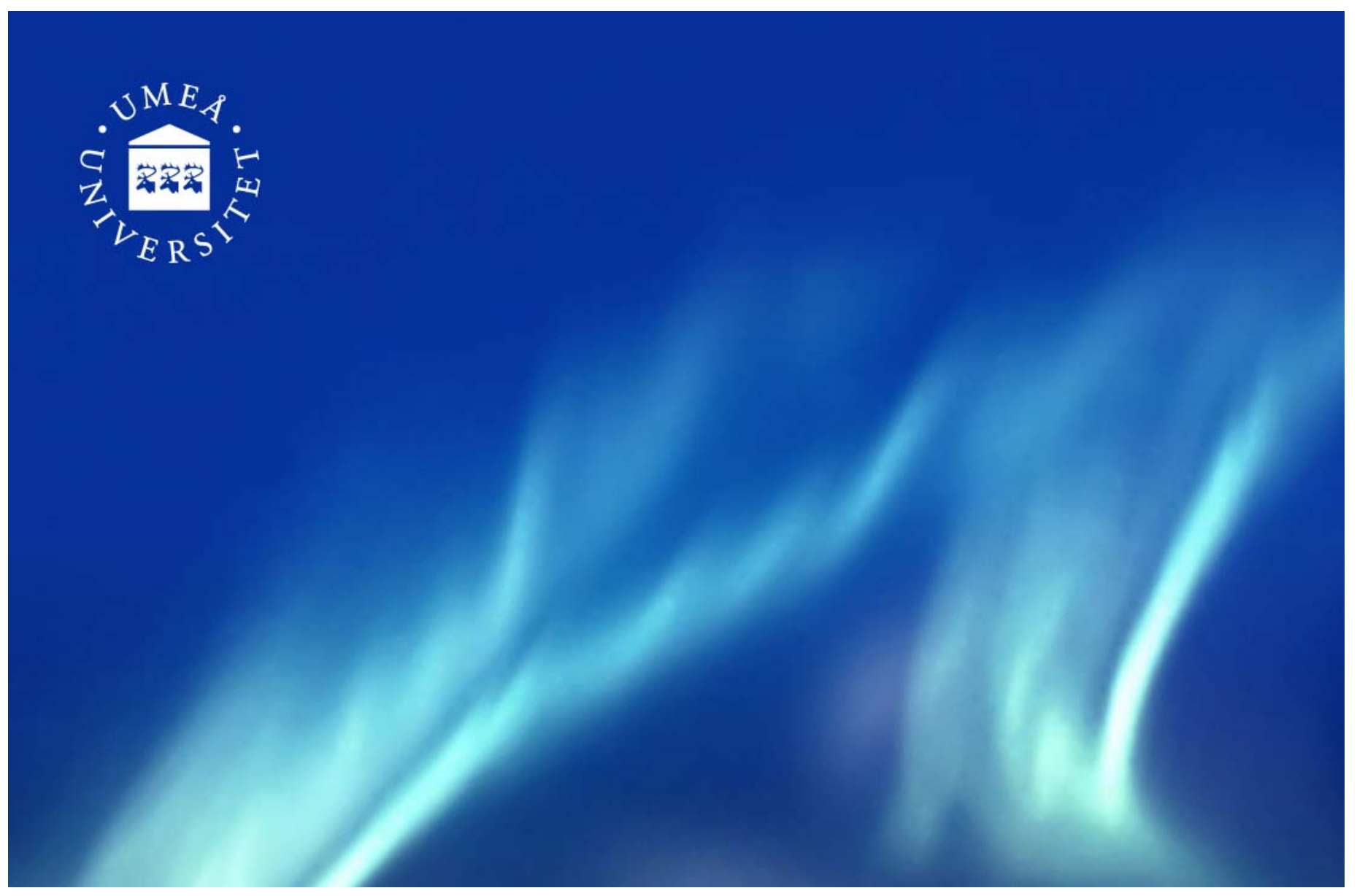

DiVA - Digitala Vetenskapliga Arkivet http://umu.diva-portal.org

This is a book chapter published in The Role of Saliva Cortisol Measurement in Health and Disease, ed. Margareta Kristenson, Peter Garvin, Ulf Lundberg.

Citation for the published paper:

Anne Helene Garde, Berndt Karlsson, Åse Marie Hansen, Roger Persson, Torbjörn Åkerstedt Sleep and Salivary Cortisol

The Role of Saliva Cortisol Measurement in Health and Disease, 2012, p. 116-128

URL: http://dx.doi.org/10.2174/978160805342111201010116 
CHAPTER 6

\title{
Sleep and Salivary Cortisol
}

\section{Anne Helene Garde ${ }^{1, *}$, Berndt Karlson ${ }^{2}$, Åse Marie Hansen ${ }^{3}$, Roger Persson ${ }^{4}$ and Torbjörn Åkerstedt ${ }^{5}$}

${ }^{1}$ Senior reseacher at the National Research Centre for the Working Environment, Lersø Parkalle 105, 2100 København Ø, Copenhagen, Denmark; ${ }^{2}$ Doctor in Occupational and Environmental Medicine,at the Department of Public Health and Clinical Medicine, Umea University, Sweden; ${ }^{3}$ Senior reseacher at the National Research Centre for the Working Environment, Copenhagen, Denmark; ${ }^{4}$ Senior reseacher at the National Research Centre for the Working Environment, Copenhagen, Denmark and ${ }^{5}$ Professor at the Stress Research Institute, Stockholm University, Stockholm, Sweden

\begin{abstract}
The aim of the present chapter was to analyze whether measures of cortisol in saliva were associated with measures of sleep and to explore if divergent results were related to underlying differences in theoretic assumptions and methods. Measures of sleep quality included sleep duration, overall sleep quality, difficulty falling asleep, disturbed sleep, and sleep deprivation. Twenty-three papers were found to fulfil the inclusion criteria. Cortisol measures were grouped into single time points at different times during the day, deviations at different time periods during the day, reactivity and recovery after a standardized laboratory test, area under the curve and response to dexamethasone test. A large proportion of the studies included showed nonsignificant findings, which, in several cases, may be a result of low power. The most consistent results were a positive association between sleep duration and single measures of salivary cortisol at awakening, which was observed in 3 studies. In these studies, sleep duration was also associated with low evening cortisol levels, steep diurnal deviation of cortisol and/or high area under the curve. Together these findings suggest that longer sleep duration is related to a more dynamic cortisol secretion. Two of the 6 studies on disturbed or restless sleep showed relations to flat diurnal deviation and low laboratory stress test reactivity. This to some extent corroborates the findings on sleep duration. However, the many nonsignificant findings as well as the theoretical and methodological differences (e.g., heterogeneity in measures) complicate comparisons. Conflicting results may be at least partially due to differences in methods and underlying assumptions.
\end{abstract}

Keywords: Salivary cortisol, sleep, sleep quality, sleep duration, sleep deprivation, difficulty falling asleep, single time point measures, deviations measures, area under the curve, dexamethasone.

\section{INTRODUCTION}

The stress response can be described as an increase in arousal in response to a real or anticipated perturbation of homeostasis [1]. The Hypothalamus-Pituitary-Adrenal Cortex (HPA) axis is one of the main stress systems with cortisol as a main actor [2,3]. The underlying anatomy of the stress response is closely interconnected with the anatomy that regulates sleep and wakefulness $[4,5]$. Emotional and cognitive arousal may therefore provide inputs that override the normal circadian and homeostatic processes that otherwise govern sleep and wakefulness in normally healthy humans $[4,6]$. The interconnectedness also makes sleep a potent factor that may modulate most components of the endocrine system [6]. To summarize, there is a possible bidirectionality between stress and sleep.

Cortisol levels have a circadian peak early in the morning, show a decline throughout the day and are near the limits of detection in the late evening [6]. The secretion of cortisol is inhibited at sleep onset, and during the early part of the sleep period, and cortisol concentrations continue to decrease until a few hours before normal waking time when they start to rise again [6-8].

In experimental studies, induced sleep deprivation lead to higher cortisol concentrations the subsequent

*Address correspondence to Anne Helene Garde: Senior reseacher at the National Research Centre for the Working Environment, Lersø Parkallé 105, 2100 København Ø, Copenhagen, Denmark; Tel: +45 391652 00; Fax: +45 391652 01; E-mail: ahg@nrcwe.dk 
evening [8] and HPA axis hormones such as cortisol-releasing hormone had a negative effect on sleep quality with increased episodes of rapid eye movement sleep and inhibited Slow-Wave Sleep (SWS). In contrast, cortisol has been shown to promote SWS [9].

Although the theoretic and empirical evidence of a close interconnectedness between sleep and HPA axis hormones is strong, there are still several unknowns with regard to understanding the interplay between stress reactions and sleep. As in other areas of stress research, findings have been disparate on these interactions.

\begin{abstract}
AIM
The aim of the present chapter was to analyze whether measures of cortisol in saliva were associated with measures of sleep and to see if possible divergent results were functions of differences in assumptions made and methods used.
\end{abstract}

\title{
METHOD
}

In a first step, an online search of the NCBI PubMed database (National Library of Medicine, National Institutes of Health, Bethesda, MD, USA-http://www.ncbi.nlm.nih.gov/PubMed) was conducted. The search covered the time period up to October 1, 2009. The search terms were "sleep AND (saliva OR salivary) AND cortisol". One hundred and eight-eight papers were found after limiting the search to papers written in English and studies on humans. Of these, 69 were selected for further scrutiny based on the titles and abstracts. They were supplemented with hand searches. In this step, studies were only included in this review if the study group comprised healthy adults and the study included specific statistical analyses of the association between sleep and cortisol.

Measures of sleep quality included (1) sleep duration, (2) overall sleep quality, (3) difficulty falling asleep, (4) disturbed sleep, (5) premature awakening, and (6) sleep deprivation. Sleep duration is a well-defined measure of the number of hours a person sleeps. It may be assessed from self-reports, actigraphy, or polysomnography (PSG). Reports on sleep quality such as ease of awakening, sleep efficiency, and sufficient sleep by use of questionnaire, logbook or actigraphy were all considered as indicators of overall sleep quality. Sleep quality may be related to sleep problems and divided into categories related to different parts of the sleep: difficulty falling asleep, disturbed sleep (difficulties maintaining sleep), and premature awakening. Difficulty falling asleep covered ease of sleep (inverted), speed of sleep onset (inverted), sleep latency, but not sleep onset, and time of falling asleep. Disturbed sleep covered restless sleep, nocturnal awakenings, time awake after sleep onset, number of microarousals during the night, and number of wake periods after sleep onset. In studies of sleep deprivation participants are actively kept awake.

In the following analyses findings were considered significant if $\mathrm{p}$-values were $<0.05$. As most of the studies had small numbers and seemingly low statistical power, we also included marginally significant results $(0.05<\mathrm{p}<0.10)$ denoted by arrows in parentheses in Table $\mathbf{1}$.

\section{RESULTS}

In total 23 papers fulfilled the inclusion criteria. A brief summary of the results (indicated as arrows denoting positive associations, or negative association and zero for a nonsignificant finding) are presented in Table 1. More detailed information on study design, statistical approach, main results, and discussion for each of the 23 papers is presented in Table 2 .

Results are presented for each sleep measure. Cortisol measures were grouped as follows. Single time points at: a1, awakening; a2, morning; a3, midday; a4, evening; a5, all day. Deviations during: b1, morning; b2, midday; b3, morning to evening; b4, laboratory test. Area Under the Curve (AUC): c1, morning (increase/ground). Suppression test: d, response to dexamethasone (DST). No studies were found for premature awakening.

\section{Sleep Duration}

Thirteen papers were found to test the association between salivary cortisol and sleep duration [10-22]. In the 13 papers there were 37 analyses on relationships between measures of salivary cortisol and sleep 
duration. The proportion of significant relationships were 4/16 (25\%) for single time points, $6 / 12$ (50\%) for deviations, $2 / 8(25 \%)$ for AUC and 0/1 (0\%) for dexamethasone test.

The most consistent results were a positive association between sleep duration and a single measure of salivary cortisol at awakening found in 3 studies [19-21]. In these studies, sleep duration was also associated with low evening cortisol levels [19], steep diurnal deviation of cortisol [19, 20], and with high AUC [21].

In 7 studies the authors failed to find any statistically significant associations between single measures of cortisol and sleep duration [11, 12, 15-17, 22]. The size of these studies was, in general, very small.

The association between sleep duration measures and deviations in cortisol measures was investigated in 7 studies. Morning deviations in cortisol concentrations were found to be positively associated with sleep length in an experimental study of 16 young people (8 morningness and 8 eveningness) using PSG [16]. In 2 ambulatory studies with more than 200 participants $[10,14]$ and a study of 2761 civil servants using selfreports negative associations to morning deviation in cortisol concentrations [20] were found.

Two studies showed a positive association between self-reported sleep duration and diurnal deviation of cortisol $[19,20]$. In 4 other studies, no significant associations were found $[11,12,15,22]$, although tendencies were observed in 1 [22].

Morning AUC was the only AUC investigated in relation to sleep duration [12, 13, 18, 21, 22]. One study, a case study with 50 days of sampling, showed a positive relationship. In contrast, 1 study, which used an insomnia scale and defined sleep duration as "more than six hours sleep", showed a negative relationship. Two out of 4 studies had only nonsignificant findings.

\section{Overall Sleep Quality}

Associations between sleep quality and measures of salivary cortisol were assessed in 8 studies $[11,12,15,17$, $19,21,23,24]$. In the 8 papers there were 28 analyses on relationships between measures of salivary cortisol and overall sleep quality. The proportion of significant relationships was $5 / 21(24 \%)$ for single time points, $1 / 5$ $(20 \%)$ for deviations, and 0/2 (0\%) for AUC. Sleep quality was measured mainly by use of self-reports, but also PSG [13].

The most consistent pattern, a positive association to a single measure at awakening [11] or in the morning $[17,23]$, was observed in 3 studies. However, 5 other studies found no associations with a single morning or awakening cortisol measure [12, 15, 19, 21, 24]. In 4 studies, sleep quality was examined in relation to single measures in the afternoon or an evening measure; no associations were found [11, 15, 19, 24]. No significant associations were seen for sleep quality and deviations in cortisol concentrations [12, 19, 24].

One study found a positive relationship between stress reactivity and sleep quality measured as sleep efficiency by actigraphy, but not by self-reports [15]. One study examined associations between sleep quality and morning AUC, and found no significant relationship [21].

\section{Difficulty Falling Asleep}

Three studies assessed a total of 10 associations between salivary cortisol and difficulty falling asleep [15, $23,25]$. The proportion of significant relationships was $0 / 5(0 \%)$ for single time points, $2 / 3(67 \%)$ for deviations, and 1/2 (50\%) for AUC. Difficulty falling asleep was assessed by use of actigraphy and selfreports (ease of sleep (inverted), speed of sleep onset (inverted), sleep latency, and time to fall asleep). The studies all used different types of cortisol measures.

Only 1 of the 3 studies reported significant associations, and the results were mixed [25]. In the same study the association between self-reported difficulty falling asleep in terms of ease of sleep was positively related to slope, whereas speed of sleep onset was negatively related [25]. High self-reported difficulty falling asleep was related to high AUC morning [25]. No other significant associations were observed between self-reported ease of sleep and measures of cortisol [15, 23, 25]. 


\section{Disturbed Sleep/Restless Sleep}

Disturbed or restless sleep was examined in 6 studies $[11,12,15,20,21,26]$ analyzing a total of 22 relationships. The proportion of significant relationships was 3/13 (23\%) for single time points, 4/7 (57\%) for deviations, and 0/2 (0\%) for AUC. Disturbed sleep was assessed as the number of microarousals during the night using PSG, forced awakening, actigraphy, and self-reports (restless sleep, nocturnal awakenings, time awake after sleep onset, and number of wake periods after sleep onset).

Four studies included associations with a single cortisol measure at awakening or in the morning: 1 found a positive association with the number of microarousals [12], 1 found a negative association with selfreported frequency of nightly awakenings, but no association with self-reported wake time after sleep onset [11], and 2 found no associations [20,21]. No associations were observed for single measures of cortisol later in the day $[11,15]$.

One study investigated the relation between disturbed sleep and diurnal deviation and found a negative association [20]. No significant findings were seen in the 3 studies that investigated the relationship between morning deviations of cortisol and disturbed sleep in terms of nightly microarousals[12], forced awakenings [26], and sleep disturbance [20]. One study investigated the effect of disturbed sleep the night before a laboratory stress test, and found negative associations with reactivity [15].

AUC in the morning was tested in relation to disturbed sleep on a day to day basis in a case study with 50 days of sampling; and no significant associations were found [21].

\section{Sleep Deprivation}

Six studies investigated a total of 8 associations between sleep deprivation and measures of salivary cortisol with mixed results [27-32]. The proportion of significant relationships was $2 / 5(40 \%)$ for single time points, $1 / 3(33 \%)$ for deviations, and $0 / 0(0 \%)$ for AUC. The studies used either 1 night of sleep deprivation [28$30,32]$ or 5-6 nights of only $4 \mathrm{~h}$ sleep [27, 31].

In 1 study sleep restriction was associated with increased concentrations of cortisol in the evening and smaller decline in cortisol during the afternoon [27]. In another study it was found that cortisol concentrations were higher in the afternoon after sleep deprivation [29]. In 4 studies using cortisol concentrations in the morning, evening, and during the day following sleep deprivation, no associations were observed [28, 30-32].

Table 1: Summary of main findings of associations between measures salivary cortisol and studied domains sorted by year of publication

\begin{tabular}{|c|c|c|c|c|c|c|c|c|c|c|c|c|c|c|c|c|c|}
\hline \multirow[t]{2}{*}{ References } & \multirow[t]{2}{*}{ Year } & \multirow[t]{2}{*}{ Exposure } & \multirow[t]{2}{*}{$\begin{array}{l}\text { Awakening } \\
\text { time }\end{array}$} & \multirow[t]{2}{*}{ Design } & \multirow[t]{2}{*}{$n$} & \multirow[t]{2}{*}{$\mathrm{M} / \mathrm{W}$} & \multicolumn{5}{|c|}{$\begin{array}{l}\text { Single time points (or } \\
\text { sum/mean of two or more } \\
\text { time points) }\end{array}$} & \multicolumn{4}{|c|}{\begin{tabular}{|l} 
Deviation \\
Difference/slope \\
for two or more \\
time points
\end{tabular}} & AUC & \multirow[t]{2}{*}{$\begin{array}{l}\text { Dexamethasone } \\
\text { suppression test }\end{array}$} \\
\hline & & & & & & & a1 & a2 & a3 & $\mathrm{a} 4$ & a5 & b1 & b2 & b3 & b4 & $\mathrm{c} 1$ & \\
\hline \multicolumn{18}{|c|}{\begin{tabular}{|l|} 
Sleep duration \\
\end{tabular}} \\
\hline Wüst [10] & 2000 & SR & & C-S & 509 & 190/319 & & & & & & $\downarrow$ & & & & & \\
\hline $\begin{array}{l}\text { Backhaus } \\
{[11]}\end{array}$ & 2004 & SR & & C-C & 29 & $21 / 8$ & $(\downarrow)$ & 0 & & 0 & & & & & & & \\
\hline $\begin{array}{l}\text { Ekstedt } \\
{[12]}\end{array}$ & 2004 & PSG & $\begin{array}{l}07: 00 \mathrm{~h} \pm 1 \\
\mathrm{~h}\end{array}$ & C-S & 24 & $10 / 14$ & 0 & 0 & & & & 0 & & & & & \\
\hline $\begin{array}{l}\text { Federenko } \\
{[13]}\end{array}$ & 2004 & SR & $04: 00 \mathrm{~h}$ & Exp & 49 & $0 / 49$ & & & & & & 0 & & & & 0 & \\
\hline $\begin{array}{l}\text { Schlotz } \\
{[14]}\end{array}$ & 2004 & SR & & C-S & 219 & $102 / 117$ & 0 & & & & & $\downarrow$ & & & & & \\
\hline Wright [15] & 2007 & SR/AG & & & 53 & $0 / 53$ & & & 0 & & & & & & 00 & & \\
\hline Griefahn & 2008 & PSG & & Exp & 16 & $16 / 0$ & & 0 & & & & $\uparrow^{b}$ & & & & & \\
\hline
\end{tabular}




\begin{tabular}{|l|l|l|l|l|l|l|l|l|l|l|l|l|l|l|l|l|l|l|}
\hline$[16]$ & & & & & & & & & & & & & & & & & & \\
\hline $\begin{array}{l}\text { Gustafsson } \\
{[17]}\end{array}$ & 2008 & SR & 25 & $13 / 12$ & & 00 & & & & & & & & \\
\hline $\begin{array}{l}\text { Liberzon } \\
{[18]^{\circ}}\end{array}$ & 2008 & SR & & Pros & 31 & $13 / 18$ & & & & & & & & & & 0 & 0 & \\
\hline Hsiao [19] & 2009 & SR & $\begin{array}{l}06.65 \mathrm{~h}, \\
\text { SD } 1.3\end{array}$ & C-S & 106 & $35 / 71$ & $\uparrow$ & & & $\downarrow$ & & & $\uparrow$ & & & \\
\hline $\begin{array}{l}\text { Kumari } \\
{[20]}\end{array}$ & 2009 & SR & $\begin{array}{l}06: 13- \\
07: 44 \mathrm{~h}\end{array}$ & C-S & $\begin{array}{l}275 \\
1\end{array}$ & $?$ & $\uparrow$ & & & & & $\downarrow$ & & $\uparrow$ & & & & \\
\hline Stalder [21] & 2009 & SR & & C & 1 & $1 / 0$ & $\uparrow$ & & & & & & & & 0 & $\uparrow$ & \\
\hline $\begin{array}{l}\text { Vreeburg } \\
{[22]}\end{array}$ & 2009 & SR & $\begin{array}{l}07: 20, \text { SD } \\
1.1\end{array}$ & C-S & 491 & $199 / 292$ & & 0 & & 0 & & $(\downarrow)$ & $(\downarrow)$ & & $\downarrow$ & 0 & 0 \\
\hline
\end{tabular}

Overall sleep quality

\begin{tabular}{|c|c|c|c|c|c|c|c|c|c|c|c|c|c|c|}
\hline Bailey [23] & 1991 & SR & & C-S & 20 & $16 / 4$ & & $\uparrow *$ & & & & & & \\
\hline $\begin{array}{l}\text { Backhaus } \\
{[11]}\end{array}$ & 2004 & SR & & $\mathrm{C}-\mathrm{C}$ & 29 & $21 / 8$ & $\uparrow \uparrow$ & 00 & & 00 & & & & \\
\hline $\begin{array}{l}\text { Ekstedt } \\
{[12]}\end{array}$ & 2004 & PSG & $\begin{array}{l}07: 00 \mathrm{~h} \\
\pm 1 \mathrm{~h}\end{array}$ & C-S & 24 & $10 / 14$ & 0 & 0 & & & 0 & & & \\
\hline Wright [15] & 2007 & SR & & & 53 & $0 / 53$ & & & 00 & & & & $\uparrow 0$ & \\
\hline $\begin{array}{l}\text { Gustafsson } \\
{[17]}\end{array}$ & 2008 & SR & & C-S & 25 & $13 / 12$ & & $\uparrow \uparrow 00$ & & & & & & \\
\hline $\begin{array}{l}\text { Dahlgren } \\
{[24]}\end{array}$ & 2009 & SR & & Pros & 14 & $8 / 6$ & 0 & 0 & & 0 & 0 & & & \\
\hline Hsiao [19] & 2009 & SR & $\begin{array}{l}06.65 \mathrm{~h}, \\
\text { SD } 1.29\end{array}$ & C-S & 106 & $35 / 71$ & 0 & & & 0 & & 0 & & \\
\hline Stalder [21] & 2009 & SR & & $\mathrm{C}$ & 1 & $1 / 0$ & 0 & & & & & & & 0 \\
\hline
\end{tabular}

Difficulty falling asleep

\begin{tabular}{|l|l|l|l|l|l|l|l|l|l|l|l|l|l|l|l|l|l|}
\hline Bailey [23] & 1991 & SR & & C-S & 20 & $16 / 4$ & & 00 & & & & & & & & & \\
\hline Wright [15] & 2007 & AG & & & 53 & $0 / 53$ & & & 0 & & & & & & 0 & & \\
\hline $\begin{array}{l}\text { Lasikiewicz } \\
{[25]}\end{array}$ & 2008 & SR & & C-S & 147 & $68 / 79$ & & & & & 00 & & & $\downarrow \uparrow$ & & $\downarrow 0$ \\
\hline
\end{tabular}

[25]

\begin{tabular}{|c|c|c|c|c|c|c|c|c|c|c|c|c|c|c|c|c|c|c|}
\hline \multicolumn{19}{|c|}{ Disturbed sleep } \\
\hline $\begin{array}{l}\text { Backhaus } \\
{[11]}\end{array}$ & 2004 & SR & & $\mathrm{C}-\mathrm{C}$ & 29 & $21 / 8$ & $\downarrow 0$ & 00 & & 00 & & & & & & & & \\
\hline $\begin{array}{l}\text { Ekstedt } \\
{[12]}\end{array}$ & 2004 & PSG & $\begin{array}{l}7 \mathrm{AM} \pm 1 \\
\mathrm{~h}\end{array}$ & $\mathrm{C}-\mathrm{S}$ & 24 & $10 / 14$ & $\uparrow$ & $\uparrow$ & & & & 0 & & & & & & \\
\hline $\begin{array}{l}\text { Dettenborn } \\
\text { [26] }\end{array}$ & 2007 & $\begin{array}{l}\text { Forced } \\
\text { awake }\end{array}$ & & Exp & 13 & $0 / 13$ & & & & & & 0 & & & & & & \\
\hline Wright [15] & 2007 & AG & & & 53 & $0 / 53$ & & & 000 & & & & & & $\downarrow \downarrow \downarrow$ & & & \\
\hline $\begin{array}{l}\text { Kumari } \\
{[20]}\end{array}$ & 2009 & SR & $6: 13-7: 44$ & C-S & $\begin{array}{l}275 \\
1\end{array}$ & & 0 & & & & & 0 & & $\downarrow$ & & & & \\
\hline Stalder [21] & 2009 & SR & & $\mathrm{C}$ & 1 & $1 / 0$ & 0 & & & & & & & & & 0 & 0 & \\
\hline \multicolumn{19}{|c|}{ Sleep deprivation } \\
\hline $\begin{array}{l}\text { Spiegel } \\
{[27]}\end{array}$ & 1999 & $\begin{array}{l}\text { Sleep } \\
\text { restricted }\end{array}$ & & Exp & 11 & $11 / 0$ & & & & $\uparrow$ & & & $\downarrow$ & & & & & \\
\hline Heiser [28] & 2000 & Forced & & Exp & 10 & $10 / 0$ & & & & & & & & 0 & & & & \\
\hline Goh [29] & 2001 & One night & & Exp & 14 & $0 / 14$ & & & $\uparrow$ & & & & & & & & & \\
\hline Pagani [30] & 2009 & One night & & Exp & 24 & $12 / 12$ & & 0 & & 0 & & & & & & & & \\
\hline $\begin{array}{l}\text { Van } \\
\text { Leeuwen } \\
{[31]}\end{array}$ & 2009 & Restricted & & Exp & 19 & $19 / 0$ & & & & & 0 & & & & & & & \\
\hline $\begin{array}{l}\text { Birchler- } \\
\text { Pedross } \\
{[32]}\end{array}$ & 2009 & $40 \mathrm{~h}$ & & Exp & 32 & $16 / 16$ & & & & & & & & 0 & & & & \\
\hline
\end{tabular}

Abbreviations: a1, awake; a2, morning; a3, midday; a4, evening; a5, all day; b1, morning; b2, midday; b3, morning to evening; b4, laboratory test reactivity/recovery; c1, morning increase/ground; AG, actigraphy; AUC, Area under the curve (increase vs ground) ; , case; C-C, case-control; C-S, cross-sectional; Exp, experimental; M, men; Pros, prospective; PSG, polysomnography; SR, selfreported; $\mathrm{W}$, women. $\uparrow$ indicates that the slope is steeper.

${ }^{\text {a }}$ Sleep length $>6 \mathrm{~h}$.

${ }^{\mathrm{b}}$ Significant finding only in evening types. 
Table 2: Descriptives of the articles on salivary cortisol and sleep parameters sorted by domain of sleep parameter and year of publication

\begin{tabular}{|c|c|c|c|c|c|c|c|}
\hline References & Outcome & $\begin{array}{l}\text { Study design/group } \\
\text { characteristics }\end{array}$ & Sampling & $\begin{array}{l}\text { Laboratory method and } \\
\text { standardization in sampling }\end{array}$ & $\begin{array}{l}\text { Statistical approach for } \\
\text { cortisol measurement in } \\
\text { relation to sleep }\end{array}$ & $\begin{array}{l}\text { Statistical analysis, cortisol in } \\
\text { relation to outcome }\end{array}$ & Results on cortisol and sleep \\
\hline $\begin{array}{l}\text { Wüst } 2000 \\
{[10]}\end{array}$ & $\begin{array}{l}\text { Sleep length: } \\
\text { TST } \\
\text { Method: } \\
\text { Self-report }\end{array}$ & $\begin{array}{l}\text { Design: C-S } \\
\text { No.: } 509 \\
\text { M/W: } 190 / 319 \\
\text { Age: } 37.3(18-71) \text { years } \\
\text { Group: Healthy }\end{array}$ & $\begin{array}{l}\text { Days: } 2 \\
\text { Samples per day: } 4 \\
\text { Times for sampling: } \\
\text { Awakening, }+15,30 \text {, and } 60 \\
\text { min, } \\
\text { Setting: Ambulatory (at home) }\end{array}$ & RIA & \begin{tabular}{|l|} 
Measurement(s): \\
a1. Cortisol on awakening \\
b1. Mean increase from \\
awakening \\
c1. AUC
\end{tabular} & $\begin{array}{l}\text { Cortisol data: Continuous } \\
\text { Statistics: Pearson correlation } \\
\text { and ANOVA with repeated } \\
\text { measures }\end{array}$ & $\begin{array}{l}\text { Positive correlation between } \\
\text { sleep duration and mean cortisol } \\
\text { increase from awakening (b1) }\end{array}$ \\
\hline $\begin{array}{l}\text { Backhaus } \\
2004 \text { [11] }\end{array}$ & $\begin{array}{l}\text { Sleep length: } \\
\text { TST } \\
\text { Sleep quality: } \\
\text { PSQI, feeling of recovery } \\
\text { Disturbed sleep: } \\
\text { Frequency of nightly } \\
\text { awakenings, wake time after } \\
\text { sleep onset } \\
\text { Method: } \\
\text { Questionnaire (PSQI), } \\
\text { feeling of recovery }\end{array}$ & $\begin{array}{l}\text { Design: C-C } \\
\text { No.: } 29 \\
\text { M/W: } 21 / 8 \\
\text { Age: } 32-62 \text { years } \\
\text { Group: insomniacs } \\
(n=14) \text { and healthy } \\
\text { controls }(\mathrm{n}=15)\end{array}$ & $\begin{array}{l}\text { Days: } 7 \\
\text { Samples per day: } 3 \\
\text { Times for sampling: } \\
\text { Awakening, }+15 \text { min and } \\
\text { before going to bed } \\
\text { Setting: Ambulatory (at home) }\end{array}$ & $\begin{array}{l}\text { RIA } \\
\text { Not to use food, alcoholic } \\
\text { beverages, caffeine, fruit } \\
\text { juice, or brush teeth } 1 \mathrm{~h} \\
\text { before sampling }\end{array}$ & \begin{tabular}{|l|} 
Measurement(s): \\
All by means of same time \\
point over the 3 \\
consecutive days \\
a1. Cortisol on awakening \\
a2. Cortisol 15 min after \\
awakening \\
a4. Cortisol at bedtime
\end{tabular} & $\begin{array}{l}\text { Cortisol data: Continuous } \\
\text { Statistics: Pearson correlation } \\
\text { and ANOVAs }\end{array}$ & $\begin{array}{l}\text { Trend for negative correlation } \\
\text { between TST and cortisol at } \\
\text { awakening (a1) } \\
\text { Positive correlation between } \\
\text { sleep quality, and feeling of } \\
\text { recovery, and cortisol at } \\
\text { awakening (a1) } \\
\text { Negative correlation between } \\
\text { frequency of nightly awakenings } \\
\text { and cortisol at awakening (a1). } \\
\text { No correlation between wake } \\
\text { time after sleep onset and } \\
\text { awakening cortisol (a1) } \\
\text { No correlation between sleep } \\
\text { parameters and cortisol } 15 \text { min } \\
\text { after awakening (a2) or cortisol at } \\
\text { bedtime (a4) }\end{array}$ \\
\hline $\begin{array}{l}\text { Ekstedt } 2004 \\
{[12]}\end{array}$ & $\begin{array}{l}\text { Sleep length: } \\
\text { TST } \\
\text { Sleep quality: } \\
\text { Sleep efficiency } \\
\text { Disturbed sleep: } \\
\text { Number of arousals } \\
\text { Method: } \\
2 \text { PSG recordings carried out } \\
\text { in the subject's home (before } \\
\text { workday/ } \\
\text { day off) }\end{array}$ & $\begin{array}{l}\text { Design: C-S } \\
\text { No.: } 24 \\
\text { M/W: } 10 / 14 \\
\text { Age: } 30.5 \pm 0.5 \text { years } \\
\text { Group: High (n=12) and } \\
\text { low (n=12) burnout, } \\
\text { recruited from a Swedish } \\
\text { IT company }\end{array}$ & $\begin{array}{l}\text { Days: } 2 \\
\text { Samples per day: } 9 \\
\text { Times for sampling: } \\
\text { Awakening, }+15,30 \text {, and } 60 \\
\text { min, } 11: 00,15.00,19: 00,21: 00 \\
\text { h and bedtime } \\
\text { Setting: Ambulatory. Saliva } \\
\text { collected at day after the PSG }\end{array}$ & $\begin{array}{l}\text { RIA } \\
\text { No current smokers, non- } \\
\text { sedentary lifestyle and } \\
\text { moderate alcohol intake }\end{array}$ & \begin{tabular}{|l|} 
Measurement(s): \\
a1. Single awakening \\
sample \\
a2. Awakening cortisol as \\
a mean morning value of 4 \\
samples, at awakening, 15, \\
30,60 min post awakening \\
b1. Deviation morning \\
value (CAR (difference $0-$ \\
60 min)
\end{tabular} & $\begin{array}{l}\text { Cortisol data: Log transformed } \\
\text { Statistics: Stepwise multiple } \\
\text { regression analyses. Pearson } \\
\text { correlation coefficient } \\
\text { Confounders: }\end{array}$ & $\begin{array}{l}\text { No association between total } \\
\text { sleep time and awakening } \\
\text { cortisol or mean cortisol within } \\
60 \text { min after awakening } \\
\text { No association between sleep } \\
\text { efficiency and cortisol } \\
\text { More nightly arousals were } \\
\text { associated with higher } \\
\text { awakening cortisol and mean } \\
\text { cortisol within } 60 \text { min after } \\
\text { awakening } \\
\text { No association between any sleep } \\
\text { measure and morning deviation }\end{array}$ \\
\hline $\begin{array}{l}\text { Federenko } \\
2004[13]\end{array}$ & $\begin{array}{l}\text { Sleep duration: } \\
\text { TST } \\
\text { Method: } \\
\text { Self-report }\end{array}$ & $\begin{array}{l}\text { Design: Exp } \\
\text { No.: } 49 \\
\text { M/W: } 0 / 49 \\
\text { Age: nurses: } 40.3 \text { years, } \\
\text { students: } 25 \text { years } \\
\text { Group: Nurses working } \\
\text { shifts }(n=18) \text { and students } \\
\text { with regular sleep cycle } \\
(n=31)\end{array}$ & \begin{tabular}{|l} 
Days: 2 \\
Samples per day: 4 \\
Times for sampling: \\
Awakening, $+30,45$ and 60 \\
min \\
Setting: Nurses: collected 1st \\
and 2nd day of 3 different \\
shifts. Students: after early \\
evening nap on 2 days
\end{tabular} & $\begin{array}{l}\text { RIA } \\
\text { Not to smoke, eat and drink } \\
\text { just water in the first hour } \\
\text { after awakening, not to } \\
\text { brush teeth, avoid } \\
\text { microinjuries in oral cavity }\end{array}$ & $\begin{array}{l}\text { Measurement(s): } \\
\text { b1. Mean increase from } \\
\text { awakening } \\
\text { c1. } \text { AUC }_{\text {ground }}\end{array}$ & $\begin{array}{l}\text { Cortisol data: } \\
\text { Statistics: Person's correlations } \\
\text { Confounders: Oral } \\
\text { contraceptives }\end{array}$ & $\begin{array}{l}\text { No correlation between sleep } \\
\text { duration and mean increase from } \\
\text { awening or } \mathrm{AUC}_{\text {ground }}\end{array}$ \\
\hline
\end{tabular}




\begin{tabular}{|c|c|c|c|c|c|c|c|}
\hline $\begin{array}{l}\text { Schlotz } 2004 \\
\text { [14] }\end{array}$ & $\begin{array}{l}\text { Sleep length: } \\
? \\
\text { Method: } \\
?\end{array}$ & $\begin{array}{l}\text { Design: C-S } \\
\text { No.: } 219 \\
\text { M/W: } 102 / 117 \\
\text { Age: 48.6 (24-83) years } \\
\text { Group: Healthy }\end{array}$ & $\begin{array}{l}\text { Days: } 7 \text { consecutive } \\
\text { Samples per day: } 4 \\
\text { Times for sampling: } \\
\text { Awakening, }+15,30 \text {, and } 60 \\
\text { min, } \\
\text { Setting: Ambulatory (at home) }\end{array}$ & RIA & $\begin{array}{l}\text { Measurement(s): } \\
\text { a1. Cortisol on awakening } \\
\text { b1. Mean increase from } \\
\text { awakening }\end{array}$ & $\begin{array}{l}\text { Cortisol data: Continuous } \\
\text { Statistics: ANOVA with } \\
\text { repeated measures }\end{array}$ & $\begin{array}{l}\text { No association between sleep } \\
\text { duration and cortisol on } \\
\text { awakening (a1) } \\
\text { Positive association between } \\
\text { sleep duration and mean cortisol } \\
\text { increase from awakening (b1) }\end{array}$ \\
\hline $\begin{array}{l}\text { Wright } 2007 \\
{[15]}\end{array}$ & $\begin{array}{l}\text { Sleep duration: } \\
\text { TST (actigraphy and self- } \\
\text { reports) } \\
\text { Sleep quality: } \\
\text { Sleep quality and sleep } \\
\text { efficiency } \\
\text { Difficulty falling asleep: } \\
\text { Sleep latency } \\
\text { Disturbed sleep: } \\
\text { Wake up \%, minutes awake, } \\
\text { number of wake periods } \\
\text { Method: } \\
\text { Actigraph and sleep log } \\
\text { (Pitsburgh sleep diary) over } \\
7 \text { days }\end{array}$ & $\begin{array}{l}\text { Design: C-S } \\
\text { No.: } 53 \\
\text { M/W: } 0 / 53 \\
\text { Age: } 37.3( \pm 9.9) \text { years } \\
\text { Group: Healthy }\end{array}$ & $\begin{array}{l}\text { Days: } 1 \\
\text { Samples per day: } 4 \\
\text { Times for sampling: Base line } \\
\text { cortisol before stress test (14:00 } \\
\text { h), post test, }+30 \text { min and } 45 \\
\text { min post test } \\
\text { Setting: Laboratory with stress } \\
\text { test }\end{array}$ & $\begin{array}{l}\text { Immunoassay } \\
\text { After stress test the } \\
\text { participants were asked to } \\
\text { relax and read general } \\
\text { interest magazines }\end{array}$ & $\begin{array}{l}\text { Measurement(s): } \\
\text { a3. Single measure at } \\
\text { baseline }(14: 00 \mathrm{~h}) \\
\text { b4. Reactivity to test }\end{array}$ & $\begin{array}{l}\text { Cortisol data: Logarithmic } \\
\text { (base 10) } \\
\text { Statistics: Pearson's } \\
\text { correlations, univariate } \\
\text { analysis and partial } \\
\text { correlations adjusting for } \\
\text { baseline cortisol }\end{array}$ & \begin{tabular}{|l} 
Positive association between \\
sleep efficiency (actigraph) and \\
cortisol reactivity to test \\
Negative association between all \\
3 disturbed sleep and cortisol \\
reactivity to test \\
No association between TST, \\
sleep latency, self-reports of sleep \\
quality and length and cortisol \\
reactivity to test \\
No association between actigraph \\
measures and baseline cortisol \\
$(14: 00 \mathrm{~h})$
\end{tabular} \\
\hline $\begin{array}{l}\text { Greifahn } \\
2008 \text { [16] }\end{array}$ & $\begin{array}{l}\text { Sleep duration: } \\
\text { TST } \\
\text { Method: } \\
\text { PSG }\end{array}$ & $\begin{array}{l}\text { Design: Exp } \\
\text { No.: } 16 \\
\text { M/W: } 16 / 0 \\
\text { Age: } 19-27 \text { years } \\
\text { Group: morningness } \\
(\mathrm{n}=8) \text {, eveningness }(\mathrm{n}=8)\end{array}$ & $\begin{array}{l}\text { Days: } 6 \text { days (?) } \\
\text { Samples per day: } 2 \\
\text { Times for sampling: 7:00 h, } \\
\text { +30 min. Only those when } \\
\text { wakeup is after } 06: 50 \mathrm{~h} \\
\text { Setting: Laboratory }\end{array}$ & $\begin{array}{l}\text { LIA (IBL) } \\
\text { No smoking, no teeth } \\
\text { brushing prior sampling }\end{array}$ & $\begin{array}{l}\text { Measurement(s): } \\
\text { a2. Single measures at } \\
07: 00 \mathrm{~h} \\
\text { b1. Deviation morning } \\
\text { concentration, at 07:00 } \mathrm{h} \\
\text { and } 30 \text { min later }\end{array}$ & $\begin{array}{l}\text { Cortisol data: Continous? } \\
\text { Statistics: ANCOVA with } \\
\text { repeated measures correlation } \\
\text { Confounders: }\end{array}$ & \begin{tabular}{|l|} 
TST had positive association \\
with b1 after night sleep \\
TST not associated with cortisol \\
at awakening (a2)
\end{tabular} \\
\hline $\begin{array}{l}\text { Gustafsson } \\
2008 \text { [17] }\end{array}$ & $\begin{array}{l}\text { Sleep duration: } \\
\text { Sleep length } \\
\text { Sleep quality: } \\
\text { Sufficient sleep, generally } \\
\text { difficulties sleeping because } \\
\text { of work } \\
\text { Method: } \\
\text { Questionnaire }\end{array}$ & $\begin{array}{l}\text { Design: C-S } \\
\text { No.: } 25 \\
\text { M/W: } 13 / 12 \\
\text { Age: } 24-62 \text { years } \\
\text { Group: White collar } \\
\text { workers }\end{array}$ & $\begin{array}{l}\text { Days: } 2 \\
\text { Samples per day: } 6 \\
\text { Times for sampling: } 15-30 \mathrm{~min} \\
\text { after awakening and every } 2 \mathrm{~h} \\
\text { until 20:00 } \mathrm{h} \\
\text { Setting: Ambulatory }\end{array}$ & $\begin{array}{l}\text { RIA } \\
\text { All participants were asked } \\
\text { to rise and go to bed at the } \\
\text { same times during days of } \\
\text { measurement }\end{array}$ & $\begin{array}{l}\text { Measurement(s): } \\
\text { a2. Two measures, approx. } \\
\text { 07:00 } \mathrm{h} \text { and 09:00 h }\end{array}$ & $\begin{array}{l}\text { Cortisol data: ? } \\
\text { Statistics: Linear regression. } \\
\text { Repeated measures ANOVA } \\
\text { Confounders: }\end{array}$ & $\begin{array}{l}\text { Association between less } \\
\text { sufficient sleep (better sleep } \\
\text { quality) and higher morning } \\
\text { cortisol. (both measures) } \\
\text { No association between sleep } \\
\text { duration or difficulties sleeping } \\
\text { because of work and morning } \\
\text { cortisol }\end{array}$ \\
\hline $\begin{array}{l}\text { Liberzon } \\
2008[18]\end{array}$ & $\begin{array}{l}\text { Sleep duration: } \\
\text { Method: } \\
\text { Not mentioned in methods } \\
\text { section }\end{array}$ & $\begin{array}{l}\text { Design: Follow-up } \\
\text { No.: } 31 \\
\text { M/W: } 13 / 18 \\
\text { Age: } 18-38 \text { years } \\
\text { Group: Students }(\mathrm{n}=23) \\
\text { and science staff }(\mathrm{n}=4) \\
\text { and ships crew member } \\
(\mathrm{n}=4)\end{array}$ & $\begin{array}{l}\text { Days: } 6 \\
\text { Samples per day: } 4 \\
\text { Times for sampling: } \\
\text { Awakening, }+15,30,45 \text { min } \\
\text { Setting: Ambulatory }\end{array}$ & $\begin{array}{l}\text { RIA } \\
\text { Not to eat, drink, smoke, } \\
\text { brush teeth or rinse mouth } \\
\text { until after } 45 \text { min sample }\end{array}$ & $\begin{array}{l}\text { Measurement(s): } \\
\text { c1g. AUC with respect to } \\
\text { ground } \\
\text { cli. AUC for increase } \\
\text { (awakening response) }\end{array}$ & \begin{tabular}{|l} 
Cortisol data: \\
Statistics: \\
Confounders: Perceived stress \\
and control (Likert scale)
\end{tabular} & $\begin{array}{l}\text { No other correlation between } \\
\text { total sleep time and cortisol } \\
\text { measures in total sample }\end{array}$ \\
\hline $\begin{array}{l}\text { Hsiao } 2009 \\
{[19]}\end{array}$ & $\begin{array}{l}\text { Sleep duration: } \\
\text { Total time slept } \\
\text { Sleep quality: } \\
\text { Sleep quality last night } \\
\text { Method: } \\
\text { Questionnaire }\end{array}$ & $\begin{array}{l}\text { Design: C-S } \\
\text { No.: } 106 \\
\text { M/W: 35/71 } \\
\text { Age: } 38.5 \text { years (SD 9.7) } \\
\text { Group: } 106 \text { healthy } \\
\text { subjects (and } 126 \text { patients }\end{array}$ & $\begin{array}{l}\text { Days: } \\
\text { Samples per day: } 5 \\
\text { Times for sampling: } \\
\text { Awakening, }+45 \mathrm{~min}, 12: 00 \mathrm{~h} \text {, } \\
\text { 17:00 h, } 21: 00 \mathrm{~h} \\
\text { Setting: Ambulatory }\end{array}$ & $\begin{array}{l}\text { RIA } \\
\text { Not to brush teeth, avoid } \\
\text { oral blood contamination } \\
\text { before sampling, Not to eat } \\
45 \text { min after awakening and } \\
30 \text { min before collecting } \\
\text { samples }\end{array}$ & $\begin{array}{l}\text { Measurement(s): single } \\
\text { measures at awakening } \\
\text { and over a day } \\
\text { a1. Awakening cortisol } \\
\text { a4. Evening cortisol } \\
\text { b3. Deviation (diurnal }\end{array}$ & $\begin{array}{l}\text { Cortisol data: Natural } \\
\text { logarithm } \\
\text { Statistics: Two-level individual } \\
\text { growth curve model. (multiple } \\
\text { regression model for nested, } \\
\text { repeated data) } \\
\text { Confounders: Several }\end{array}$ & $\begin{array}{l}\text { Association between longer sleep } \\
\text { and steeper slope (b3), higher } \\
\text { awakening cortisol (a1), lower } \\
\text { cortisol in the evening (a4) } \\
\text { No association between sleep } \\
\text { quality and cortisol }\end{array}$ \\
\hline
\end{tabular}




\begin{tabular}{|c|c|c|c|c|c|c|c|}
\hline & & $\begin{array}{l}\text { with major depression - } \\
\text { not used in present } \\
\text { review) }\end{array}$ & & & \begin{tabular}{|l} 
profile) \\
\end{tabular} & $\begin{array}{l}\text { confounders are adjusted for in } \\
\text { two different models }\end{array}$ & \\
\hline $\begin{array}{l}\text { Kumari } 2009 \\
{[20]}\end{array}$ & \begin{tabular}{|l|} 
Sleep duration: \\
TST divided into 1-h \\
categories \\
Disturbed sleep: \\
Sleep disturbance \\
Method: \\
Logbook and questionnaire
\end{tabular} & $\begin{array}{l}\text { Design: C-S } \\
\text { No.: } 2751 \\
\text { M/W:? } \\
\text { Age: ? } \\
\text { Group: Whitehall }\end{array}$ & $\begin{array}{l}\text { Days: } \\
\text { Samples per day: } 5 \\
\text { Times for sampling: } \\
\text { Awakening, }+30 \mathrm{~min},+2.5,8 \text {, } \\
12 \mathrm{~h} \\
\text { Setting: Ambulatory }\end{array}$ & $\begin{array}{l}\text { Immunoassay method } \\
\text { Provide } 6 \text { samples on a } \\
\text { normal weekday }\end{array}$ & $\begin{array}{l}\text { Measurement(s): } \\
\text { a1. Single time point, } \\
\text { awake } \\
\text { b1. Deviation morning } \\
\text { profile } \\
\text { b3. Deviation morning to } \\
\text { evening (profile) }\end{array}$ & $\begin{array}{l}\text { Cortisol data: Log cortisol data } \\
\text { Statistics: Multilevel, } \\
\text { interaction term } \\
\text { Confounders: Age, sex, } \\
\text { employment grade, awakening } \\
\text { time, smoking status, waist } \\
\text { circumference }\end{array}$ & $\begin{array}{l}\text { Association between long sleep } \\
\text { duration and higher cortisol. at } \\
\text { awakening (a1) and steeper } \\
\text { diurnal slope (b3). Association } \\
\text { between long sleep duration and } \\
\text { flatter morning slope (b1) } \\
\text { Association between less } \\
\text { disturbed sleep and steeper } \\
\text { diurnal slope (b3) }\end{array}$ \\
\hline $\begin{array}{l}\text { Stalder } 2009 \\
{[21]}\end{array}$ & $\begin{array}{l}\text { Sleep duration: } \\
\text { TST } \\
\text { Sleep quality: } \\
\text { Disturbed sleep: } \\
\text { Nocturnal awakenings } \\
\text { Method: } \\
\text { Sleep log }\end{array}$ & $\begin{array}{l}\text { Design: Case study } \\
\text { No.: } 1 \\
\text { M/W: } 1 / 0 \\
\text { Age: } 27 \text { years }\end{array}$ & $\begin{array}{l}\text { Days: } 50 \text { with } 3 \text { days interval } \\
\text { Samples per day: } 4 \\
\text { Times for sampling: } \\
\text { Awakening, }+15,30 \text { and } 45 \\
\text { min } \\
\text { Setting: Ambulatory }\end{array}$ & \begin{tabular}{|l|} 
ELISA method \\
Sat for 15-30 min when \\
sampling, otherwise moved \\
freely in relation to sampling \\
times
\end{tabular} & $\begin{array}{l}\text { Measurement(s): } \\
\text { a1. Awakening } \\
\text { concentration } \\
\text { c1. AUC } C_{\text {ground }} \\
\text { c1. AUC } \text { increase }\end{array}$ & $\begin{array}{l}\text { Cortisol data: Log transform } \\
\text { Statistics: Repeated measures } \\
\text { ANOVA } \\
\text { Confounders: Alcoholic drinks } \\
\text { consumed the evening before } \\
\text { measurement day }\end{array}$ & $\begin{array}{l}\text { Positive association between } \\
\text { sleep duration and awakening } \\
\text { cortisol and } \mathrm{AUC}_{\text {ground }} \\
\text { No association between disturbed } \\
\text { sleep and sleep quality and } \\
\text { cortisol } \\
\text { No association between any sleep } \\
\text { parameters and } \mathrm{AUC}_{\text {increase }}\end{array}$ \\
\hline $\begin{array}{l}\text { Vreeburg } \\
2009[22]\end{array}$ & $\begin{array}{l}\text { Sleep duration: } \\
\text { Sleep length (more or less } \\
\text { than } 6 \mathrm{~h} \text { ) } \\
\text { Method: } \\
\text { Insomnia rating scale }\end{array}$ & $\begin{array}{l}\text { Design: C-S } \\
\text { No.: } 491 \\
\text { M/W: 199/292 } \\
\text { Age: } 43.0 \text { years } \\
\text { Group: volunteers } \\
\text { without psychopathology } \\
\text { Excl: Taking } \\
\text { antidepressants, pregnant } \\
\text { or breastfeeding, on } \\
\text { medication with } \\
\text { corticosteroids } \\
\text { P rate: } 78.3 \% \text {, with at } \\
\text { least one usable cortisol } \\
\text { measurement }\end{array}$ & $\begin{array}{l}\text { Days: } 1 \\
\text { Samples per day: } 7 \\
\text { Times for sampling: } \\
\text { Awakening, }+30,45,60 \mathrm{~min}, \\
\text { 22:00 h, } 23: 00 \mathrm{~h} \text {. Samples } \\
\text { taken more than } 5 \text { min from } \\
\text { protocol time were discarded } \\
\text { Setting: Ambulatory. Day after } \\
\text { dexamethasone } 0.5 \mathrm{mg} \text { directly } \\
\text { after sampling time at } 23: 00 \mathrm{~h}\end{array}$ & $\begin{array}{l}\text { Immunoassay method } \\
\text { When sampling no eating, } \\
\text { smoking, drinking tea or } \\
\text { coffee, or brushing teeth } 15 \\
\text { min before }\end{array}$ & $\begin{array}{l}\text { Measurement(s): } \\
\text { a2. } \\
\text { a4. } \\
\text { b1. Deviation morning } \\
\text { b3. Deviation } 23: 00 \mathrm{~h}, \\
\text { awakening time divided } \\
\text { by numbers of hours in } \\
\text { between (diurnal slope) } \\
\text { c1i. AUC } \text { increase }_{\text {morning }} \\
\text { c1g. AUC } \text { ground }_{\text {morning }} \\
\text { d. Post dexamethasone } \\
\text { (DST) }\end{array}$ & $\begin{array}{l}\text { Cortisol data: AUC, evening } \\
\text { cortisol and DST were log } \\
\text { transformed } \\
\text { Statistics: Linear mixed } \\
\text { models or linear regression } \\
\text { analysis } \\
\text { Confounders: } \\
\text { Sociodemographic factors, } \\
\text { health indicators }\end{array}$ & $\begin{array}{l}\text { Less than } 6 \mathrm{~h} \text { sleep is associated } \\
\text { with increase in CAR } \\
\text { (AUC } \mathrm{Ancreses} \\
\text { Tendency for less than } 6 \mathrm{~h} \text { sleep } \\
\text { is associated with steeper } \\
\text { morning deviation (b1) and a } \\
\text { steeper diurnal slope (b3) } \\
\text { No association with morning } \\
\text { cortisol (a2), evening cortisol } \\
\text { (a4), AUC Cround morning, or post } \\
\text { dexamethasone }\end{array}$ \\
\hline $\begin{array}{l}\text { Bailey } 1991 \\
\text { [23] }\end{array}$ & $\begin{array}{l}\text { Sleep quality: } \\
\text { Sleep quality } \\
\text { Difficulty falling asleep: } \\
\text { Sleep onset } \\
\text { Method: } \\
\text { Sleep log }\end{array}$ & $\begin{array}{l}\text { Design: C-S } \\
\text { No.: } 20 \\
\text { M/W: } 16 / 4 \\
\text { Age: } 23-39 \text { years } \\
\text { Group: morning types } \\
\text { (n=10) and evening types } \\
\text { (n=10). Recruited from } \\
\text { the general population }\end{array}$ & $\begin{array}{l}\text { Days: } 1 \\
\text { Samples per day: } 7 \\
\text { Times for sampling: Arising, } \\
+20,40,60,80,100 \text {, and } 120 \\
\text { min } \\
\text { Setting: Ambulatory }\end{array}$ & RIA & $\begin{array}{l}\text { Measurement(s): } \\
\text { a2. Single cortisol levels }\end{array}$ & $\begin{array}{l}\text { Cortisol data: Continuous } \\
\text { Statistics: } t \text {-test, Pearson } \\
\text { product-moment correlation } \\
\text { coefficients, Spearman rho } \\
\text { correlation coefficients }\end{array}$ & $\begin{array}{l}\text { Positive association between } \\
\text { sleep quality and total cortisol in } \\
\text { evening type group, but not in } \\
\text { morning type group } \\
\text { No association between sleep } \\
\text { onset and total cortisol in evening } \\
\text { or morning type group }\end{array}$ \\
\hline $\begin{array}{l}\text { Dahlgren } \\
2009[24]\end{array}$ & $\begin{array}{l}\text { Sleep quality: } \\
\text { Method: } \\
\text { Karolinska sleep diary for } 4 \\
\text { weeks }\end{array}$ & $\begin{array}{l}\text { Design: C-S } \\
\text { No.: } 14 \\
\text { M/W: } 8 / 6 \\
\text { Age: } 44 \text { years } \\
\text { Group: Office workers }\end{array}$ & $\begin{array}{l}\text { Days: } 28 \\
\text { Samples per day: } 3 \\
\text { Times for sampling: } \\
\text { Awakening, }+15 \mathrm{~min} \text {, bedtime } \\
\text { Setting: Ambulatory }\end{array}$ & $\begin{array}{l}\text { RIA } \\
\text { No food, no teeth brushing } \\
30 \text { min before saliva } \\
\text { sampling }\end{array}$ & $\begin{array}{l}\text { Measurement(s): Single } \\
\text { time points: } \\
\text { a1. Awake } \\
\text { a2. Morning } \\
\text { a4. Evening } \\
\text { b1. Deviation morning }\end{array}$ & $\begin{array}{l}\text { Cortisol data: Log data } \\
\text { Statistics: Multiple regression } \\
\text { analyses by time. ANOVA of } \\
\text { repeated measurements } \\
\text { Confounders: Work day, work } \\
\text { load, awakening time, stress at } \\
\text { bedtime, sleep quality, stress, } \\
\text { sleepiness exhaustion, self } \\
\text { related health }\end{array}$ & $\begin{array}{l}\text { No associations between sleep } \\
\text { quality and measures of cortisol }\end{array}$ \\
\hline
\end{tabular}




\begin{tabular}{|c|c|c|c|c|c|c|c|}
\hline $\begin{array}{l}\text { Lasikiewicz } \\
2008 \text { [25] }\end{array}$ & $\begin{array}{l}\text { Difficulty falling asleep: } \\
\text { Ease of sleep and speed of } \\
\text { sleep onset } \\
\text { Method: } \\
\text { Questionnaire (Leeds Sleep } \\
\text { Evaluation Questionnaire) }\end{array}$ & $\begin{array}{l}\text { Design: C-S } \\
\text { No.: } 147 \\
\text { M/W::68/79 } \\
\text { Age: :mean age } 46.2 \\
\text { years }( \pm 7,2) \\
\text { Group: volunteers }\end{array}$ & $\begin{array}{l}\text { Days: } 1(n=64) \text { or } 3(n=83) \\
\text { Samples per day: } 8 \\
\text { Times for sampling: } \\
\text { Awakening, }+15,30,45 \text { min, } \\
+3,6,9,12 \mathrm{~h} \\
\text { Setting: } \\
\text { Ambulatory }\end{array}$ & \begin{tabular}{|l|} 
Immunoassay method \\
Not to consume food or \\
drink other than water in \\
relation to sample collection. \\
Avoid teeth brushing and \\
vascular leakage
\end{tabular} & $\begin{array}{l}\text { Measurement(s): Mean of } \\
\text { same time point on } \\
\text { consecutive days } \\
\text { a5. } \\
\text { b4. Deviation evening } \\
\text { from } 45 \text { min post } \\
\text { awakening (slope) } \\
\text { c1. AUC not specified }\end{array}$ & $\begin{array}{l}\text { Cortisol data: Log transformed } \\
\text { Statistics: Pearson's } \\
\text { correlation. Cluster analysis } \\
\text { (M)ANOVA } \\
\text { Confounders: Age, gender }\end{array}$ & $\begin{array}{l}\text { Association between higher ease } \\
\text { of sleep (less difficulty falling } \\
\text { asleep) and low AUC } \\
\text { Association between high ease of } \\
\text { sleep (less difficulty falling } \\
\text { asleep) and less steep slope (b3) } \\
\text { Association between high speed } \\
\text { of sleep onset (less difficulty } \\
\text { falling asleep) and more steep } \\
\text { slope (b3) } \\
\begin{array}{l}\text { No association between ease of } \\
\text { sleep, speed of sleep onset and } \\
\text { diurnal mean (a5) }\end{array} \\
\end{array}$ \\
\hline $\begin{array}{l}\text { Dettenborn } \\
2007 \text { [26] }\end{array}$ & $\begin{array}{l}\text { Disturbed sleep: } \\
\text { Method: } \\
\text { Three experimentally } \\
\text { induced awakenings (phone } \\
\text { call). The wake up in the } \\
\text { morning was optional or set } \\
\text { up by alarm clock. No other } \\
\text { sleep registration }\end{array}$ & $\begin{array}{l}\text { Design: Exp } \\
\text { No.: } 13 \\
\text { M/W: } 0 / 13 \\
\text { Age: } 24 \text { years }\end{array}$ & $\begin{array}{l}\text { Days: } 3 \text { intervention nights }+3 \\
\text { reference nights } \\
\text { Samples per day: } 8 \text { on } \\
\text { intervention nights and } 2 \text { on } \\
\text { recovery nights } \\
\text { Times for sampling: } \\
\text { Awakening and }+15 \text { min in the } \\
\text { morning } \\
\text { Setting: Ambulatory }\end{array}$ & CLIA & $\begin{array}{l}\text { Measurement(s): } \\
\text { b1. Repeated measures }\end{array}$ & $\begin{array}{l}\text { Cortisol data: continous } \\
\text { Statistics: ANOVA and } \\
\text { ANCOVA } \\
\text { Confounders: Oral } \\
\text { contraceptives, thyroid } \\
\text { hormone }\end{array}$ & $\begin{array}{l}\text { The morning CAR after } \\
\text { disturbed nights was not different } \\
\text { from CAR on undisturbed nights } \\
\text { There was a lack of HPA axis } \\
\text { activation by forced nightly } \\
\text { awakenings }\end{array}$ \\
\hline $\begin{array}{l}\text { Spiegel } 1999 \\
{[27]}\end{array}$ & $\begin{array}{l}\text { Sleep deprivation: } \\
\text { Sleep restriction }\end{array}$ & $\begin{array}{l}\text { Design: Exp } \\
\text { No.: } 11 \\
\text { M/W: } 11 / 0 \\
\text { Age: } 18-27 \text { years } \\
\text { Group: Young healthy } \\
\text { volunteers }\end{array}$ & $\begin{array}{l}\text { Days: } 3 \\
\text { Samples per day: } 12-20 \\
\text { Times for sampling: Every } 30 \\
\text { min between } 15.00 \text { and } \\
\text { bedtime } \\
\text { Setting: Laboratory. } 3 \text { nights } \\
\text { with } 8 \mathrm{~h} \text { in bed, } 6 \text { nights with } 4 \\
\mathrm{~h} \text { in bed, and } 7 \text { nights with } 12 \mathrm{~h} \\
\text { in bed }\end{array}$ & RIA & $\begin{array}{l}\text { a4. Single evening } \\
\text { concentration } \\
\text { b2. Deviation between } \\
\text { 16:00 } \mathrm{h} \text { and } 21: 00 \mathrm{~h}\end{array}$ & $\begin{array}{l}\text { Cortisol data: Continuous } \\
\text { Statistics: ANOVA for } \\
\text { repeated measures }\end{array}$ & $\begin{array}{l}\text { Higher evening cortisol } \\
\text { concentration after sleep } \\
\text { restriction } \\
\text { Lower rate of decrease in the } \\
\text { afternoon after sleep restriction }\end{array}$ \\
\hline $\begin{array}{l}\text { Heiser } 2000 \\
{[28]}\end{array}$ & \begin{tabular}{|l|} 
Sleep deprivation: \\
3 days covered, after ordinary \\
sleep, 1 night of total sleep \\
deprivation and 1 night of \\
recovery
\end{tabular} & \begin{tabular}{|l} 
Design: Exp \\
No.: 10 \\
M/W: $10 / 0$ \\
Age: $27.4 \pm 2.8$ years \\
Group: healthy \\
volunteers
\end{tabular} & $\begin{array}{l}\text { Days: } 3 \\
\text { Samples per day: } 3 \\
\text { Times for sampling: 07:00, } \\
\text { 13:00, 19:00 h } \\
\text { Setting: Laboratory }\end{array}$ & \begin{tabular}{|l|} 
RIA \\
All intake of pineapples, \\
bananas, almonds, nuts, \\
tomatoes, vanilla, or alcohol \\
forbidden, no smokers \\
\end{tabular} & $\begin{array}{l}\text { Measurement(s): } \\
\text { b3. Diurnal profile with } 3 \\
\text { measures per day over } 3 \\
\text { days }\end{array}$ & $\begin{array}{l}\text { Cortisol data: ? } \\
\text { Statistics: ANOVA with } \\
\text { repeated measures }\end{array}$ & $\begin{array}{l}\text { No effect on salivary cortisol } \\
\text { rhythm of sleep deprivation }\end{array}$ \\
\hline $\begin{array}{l}\text { Goh } 2001 \\
{[29]}\end{array}$ & $\begin{array}{l}\text { Sleep deprivation: } \\
24 \mathrm{~h} \text { sleep deprivation or } 8 \mathrm{~h} \\
\text { sleep (control) }\end{array}$ & \begin{tabular}{|l} 
Design: Exp \\
No.: 14 \\
M/W: $14 / 0$ \\
Age: $20-30$ years \\
Group: healthy subjects, \\
military service members \\
\end{tabular} & \begin{tabular}{|l} 
Days: 2 \\
Samples per day: $3-5$ \\
Times for sampling: $08: 00$, \\
13:30, 18:00, 21:00, 24:00 (day \\
1), 08:00, 13:30, 18:00 (day 2) \\
Setting: Laboratory \\
\end{tabular} & Immunoassay & b3. Deviation, all day & $\begin{array}{l}\text { Cortisol data: Continuous } \\
\text { Statistics: Two-way ANOVA } \\
\text { with repeated measures and } \\
\text { interaction terms }\end{array}$ & $\begin{array}{l}\text { Significant interaction between } \\
\text { sleep status and time. Cortisol } \\
\text { levels at 13:30 } \mathrm{h} \text { were increased } \\
\text { after sleep deprivation }\end{array}$ \\
\hline $\begin{array}{l}\text { Pagani } 2009 \\
{[30]}\end{array}$ & $\begin{array}{l}\text { Sleep deprivation: } \\
7 \text { normal nights }+24 \mathrm{~h} \text { sleep } \\
\text { deprivation) or normal living } \\
\text { conditions (strict sleep-wake } \\
\text { schedule 23:00-07:00 h) }\end{array}$ & $\begin{array}{l}\text { Design: Exp } \\
\text { No.: } 24 \\
\text { M/W: } 12 / 12 \\
\text { Age: } 27-45 \text { years } \\
\text { Group: healthy subjects }\end{array}$ & $\begin{array}{l}\text { Days: } 8 ? \\
\text { Samples per day: } 2 \\
\text { Times for sampling: } 10.30 \text { and } \\
18.00 \mathrm{~h} \\
\text { Setting: Laboratory }\end{array}$ & RIA & $\begin{array}{l}\text { Measurement(s): } \\
\text { a2. } \\
\text { a4. }\end{array}$ & $\begin{array}{l}\text { Cortisol data: Continuous } \\
\text { Statistics: Mixed model or } \\
\text { GLM analysis. Intraclass } \\
\text { correlations } \\
\text { Confounders: }\end{array}$ & $\begin{array}{l}\text { No effect of sleep deprivation on } \\
\text { cortisol }\end{array}$ \\
\hline
\end{tabular}




\begin{tabular}{|c|c|c|c|c|c|c|c|}
\hline $\begin{array}{l}\text { Van } \\
\text { Leeuwen } \\
2009[31]\end{array}$ & $\begin{array}{l}\text { Sleep deprivation: } \\
2 \text { baseline }(8 \mathrm{~h} \text { sleep), } 5 \\
\text { nights of } 4 \mathrm{~h} \text { sleep, and } 2 \\
\text { recovery nights of } 8 \mathrm{~h} \text {. } \\
\text { Controls }(8 \mathrm{~h} \text { sleep) all nights }\end{array}$ & $\begin{array}{l}\text { Design: Exp } \\
\text { No.: } 19 \\
\text { M/W:19/0 } \\
\text { Age: } 19-29 \text { years } \\
\text { Group: } 13 \text { young healthy } \\
\text { men }+6 \text { controls } \\
\end{array}$ & $\begin{array}{l}\text { Days: } \\
\text { Samples per day: } 10 \\
\text { Times for sampling: Not } \\
\text { specified } \\
\text { Setting: Laboratory }\end{array}$ & $\begin{array}{l}\text { Competitive CLIA } \\
\text { Napping during day time } \\
\text { was not allowed, meals } \\
\text { standardized (calories and } \\
\text { time), controlled } \\
\text { illumination and room } \\
\text { temperature } \\
\end{array}$ & $\begin{array}{l}\text { Measurement(s): } \\
\text { a5. Averaged throughout } \\
\text { the day }\end{array}$ & $\begin{array}{l}\text { Cortisol data: } \\
\text { Statistics: } t \text {-tests and Wilcoxon } \\
\text { signed ranks test for not } \\
\text { normally distributed } \\
\text { differences } \\
\text { Confounders: }\end{array}$ & $\begin{array}{l}\text { No change in salivary cortisol } \\
\text { after sleep restriction }\end{array}$ \\
\hline \begin{tabular}{|l} 
Birchler- \\
Pedross 2009 \\
{$[32]$}
\end{tabular} & $\begin{array}{l}\text { Sleep deprivation: } \\
40 \mathrm{~h} \text { sleep deprivation or nap } \\
\text { protocol }\end{array}$ & $\begin{array}{l}\text { Design: Exp } \\
\text { No.: } 32 \\
\text { M/W: } 16 / 16 \\
\text { Age: } 25.0 \pm 3.3 \text { and } \\
65.0 \pm 5.5 \text { years } \\
\text { Group: Young and older } \\
\text { healthy volunteers }\end{array}$ & $\begin{array}{l}\text { Days: } 2 \\
\text { Samples per day: 5-6. Times } \\
\text { for sampling: every } 30 \mathrm{~min} \\
\text { collapsed into } 08: 00,12: 00, \\
\text { 16:00, 20:00, 24:00 } \mathrm{h}(\text { day } 1), \\
04: 00,08: 00,12: 00,16: 00, \\
\text { 20:00, 24:00 } \mathrm{h} \text { (day 2) } \\
\text { Setting: Laboratory }\end{array}$ & RIA & b3. Deviation, all day & $\begin{array}{l}\text { Cortisol data: Continuous } \\
\text { Statistics: Repeated measures } \\
\text { ANOVA with interaction } \\
\text { terms }\end{array}$ & $\begin{array}{l}\text { Significant four-way interaction } \\
\text { term (time of day, age, gender, } \\
\text { sleep pressure) in model for } \\
\text { cortisol, most likely driven by } \\
\text { time of day } \\
\text { None of the other variables were } \\
\text { significant }\end{array}$ \\
\hline $\begin{array}{l}\text { Vreeburg } \\
2009[22]\end{array}$ & $\begin{array}{l}\text { Sleep duration: } \\
\text { Sleep length (more or less } \\
\text { than } 6 \mathrm{~h} \text { ) } \\
\text { Method: } \\
\text { Insomnia rating scale }\end{array}$ & $\begin{array}{l}\text { Design: C-S } \\
\text { No.: } 491 \\
\text { M/W: } 199 / 292 \\
\text { Age: } 43.0 \text { years } \\
\text { Group: volunteers } \\
\text { without psychopatology } \\
\text { Excluded: Taking } \\
\text { antidepressants, pregnant } \\
\text { or breastfeeding, on } \\
\text { medication with } \\
\text { corticosteroids } \\
\text { P rate: } 78.3 \% \text {, with at } \\
\text { least one usable cortisol } \\
\text { measurement }\end{array}$ & $\begin{array}{l}\text { Days: } 1 \\
\text { Samples per day: } 7 \\
\text { Times for sampling: } \\
\text { Awakening, }+30,45,60 \mathrm{~min}, \\
\text { 22:00, } 23: 00 \mathrm{~h} \text {. Samples taken } \\
\text { more than } 5 \text { minutes from } \\
\text { protocol time were discarded } \\
\text { Setting: Ambulatory. Day after } \\
\text { dexamethasone } 0.5 \mathrm{mg} \text { directly } \\
\text { after sampling time at } 23: 00 \mathrm{~h}\end{array}$ & $\begin{array}{l}\text { Immunoassay method } \\
\text { When sampling no eating } \\
\text { ssmoking, drinking tea or } \\
\text { coffee, or brushing teeth } 15 \\
\text { min before }\end{array}$ & $\begin{array}{l}\text { Measurement(s): } \\
\text { a2. } \\
\text { a4. } \\
\text { b1. Deviation morning } \\
\text { b3. Deviation } 23: 00 \mathrm{~h} \mathrm{to} \\
\text { awakening time divided } \\
\text { by number of hours in } \\
\text { between (diurnal slope) } \\
\text { c1i. AUC } C_{\text {increase }} \text { morning } \\
\text { c1g. AUC } C_{\text {ground }} \text { morning } \\
\text { d. Post DST }\end{array}$ & $\begin{array}{l}\text { Cortisol data: AUC, evening } \\
\text { cortisol and DST were log } \\
\text { transformed } \\
\text { Statistics: Linear mixed } \\
\text { models or linear regression } \\
\text { analysis } \\
\text { Confounders: } \\
\text { Sociodemographic factors, } \\
\text { health indicators }\end{array}$ & $\begin{array}{l}\text { Less than } 6 \mathrm{~h} \text { sleep is associated } \\
\text { with increase in CAR } \\
\text { (AUC } \text { increses) } \\
\text { Tendency for less than } 6 \mathrm{~h} \text { sleep } \\
\text { is associated with steeper } \\
\text { morning deviation (b1) and a } \\
\text { steeper diurnal slope (b3) } \\
\text { No association with morning } \\
\text { cortisol (a2), evening cortisol } \\
\text { (a4), AUC } C_{\text {ground }} \text { morning, or post } \\
\text { dexamethasone }\end{array}$ \\
\hline
\end{tabular}

Abbreviations: AUC, Area under the curve; C-C, case-control; C-S, cross-sectional; CAR, cortisol awakening response; CLIA, chemiluminescence-assay; DST, Dexamethasone test; ELISA, Enzyme Linked Immuno-Sorbant Assay; Exp, experimental; GLM, generalized linear model; LIA, luminescence immunoassay; M, Male; PSG, polysomnography; PSQI, Pittsburgh Sleep Quality Index; P rate, Response rate; RIA, radioimmunoassay; TST, total sleep time, W, women. 


\section{DISCUSSION}

The aim of the present chapter was to analyze whether measures of cortisol in saliva were associated with measures of sleep to see if divergent results were functions of differences in theoretic assumptions made and methods used. Relatively few papers were first identified $(n=188)$, and only 23 papers met the final inclusion criteria.

The most consistent results were a positive association between sleep duration and a single measure of salivary cortisol at awakening, which was observed in 3 studies [19-21]. In these studies, sleep duration was also associated with low evening cortisol levels [19], steep diurnal deviation of cortisol [19, 20], and with high AUC [21]. Together these findings suggest that longer sleep duration is related to a more dynamic cortisol secretion.

However, long sleep duration is also associated with a lower CAR $[10,14,20]$. Since a lower CAR implies a less dynamic response these observations seems to contradict the suggestion that longer sleep duration is related to a more dynamic cortisol secretion. However, a lower CAR needs not to be mutually exclusive with a dynamic cortisol secretion as assessed by the decline in concentrations during the entire day. Indeed, a lower CAR might just reflect the diurnal rhythmicity of cortisol secretion. For example, to the extent that participants, who sleep long wake up later than usual, they are likely to take their first sample at a time when the concentrations of cortisol already have risen due to the normal diurnal rhythm. Hence, there is simply less room to obtain a high CAR as the morning value has been inflated by the underlying diurnal rhythm. However, there might also be several other explanations and this question deserves more attention in future studies.

Two of the 6 studies on disturbed sleep showed that it was associated with less diurnal deviation [20], and lower reactivity to a laboratory stress test [15]. This to some extent corroborates the findings on sleep duration. However, the many nonsignificant findings as well as the theoretic and methodological differences (e.g. heterogeneity in measures) complicate comparisons.

As expected, statistically significant associations were more often reported in studies with a large number of participants or a high sampling frequency. Among the papers included, statistically significant findings were generally seen in studies with more than 100 participants [10,14, 19, 20], or samples (case study) [21]. In the large studies, it was more common to focus on general sleep patterns, whereas the case study registered day to day variations within the same person. The results suggest that a relationship between sleep and salivary cortisol is observable both within and between subjects, but requires many observations due to high variability in both cortisol and sleep measures. The use of small study samples and non-optimal measurement procedures might lead to studies with low power and failure to detect statistically significant results.

Even if the inclusion criteria drastically reduced the number of papers, the papers included covered several different types of measures and indicators for cortisol and sleep. This heterogeneity in measures and methods appears to generate a mixed pattern of results that are also conflicting at times. For example, in the same study a positive relationship was observed between stress reactivity and sleep quality measured as sleep efficiency by actigraphy, but not by self-reports [15]. As differences between objectively measured and self-reports of sleep have been observed previously, this conflict may be partially related to the selected instrument for measurement of sleep quality [33].

There are several methodological problems in studies of sleep and saliva cortisol. Because saliva sampling requires that the person is awake, it is virtually impossible to sample saliva during sleep and to establish the relationship with different sleep stages or other processes that may occur during sleep. It is often not practically feasible in field studies to obtain direct measurements during the sleep period with, for example, PSG or endocrine measures. Even if it is technologically possible to use such measures, the procedures and commitments necessary for successful implementation have often been considered to be cumbersome and too expensive to be considered as a realistic option. Stress researchers often have to rely on less sophisticated and simpler approaches such as motion logging (e.g., actigraphy) and subjective reports of various aspects of sleep (e.g., bedtime, awakening time, and perceived quality of sleep). 
Many of the studies were not conducted primarily to evaluate the relationship between sleep and salivary cortisol. Even so, all studies included in the present chapter have used statistical tests to address the overall question about the relationship between sleep and saliva cortisol. Considering that research have been driven by many points of departures, it is not surprising that most of the result do not act in concert.

\section{CONCLUSIONS}

In total 23 studies examining sleep quality in relation to salivary cortisol measures were identified. There was a large proportion of nonsignificant findings and many operational definitions of sleep quality and cortisol secretion. Because many of the studies included were small and entailed few measurements, there is reason to believe that the nonsignificant findings partly reflect low statistical power.

The most consistent results were our observation of a positive association between sleep duration and a single measure of salivary cortisol at awakening, which was observed in 3 studies. In these studies, sleep duration was also associated with low evening cortisol levels, steep diurnal deviation of cortisol, and/or with high AUC. Together these findings suggest that longer sleep duration is related to a more dynamic cortisol secretion. Two of the 6 studies on disturbed or restless sleep showed a relationship with flat diurnal deviation and low laboratory stress test reactivity. This to some extent corroborates the findings regarding sleep duration. However, the many nonsignificant findings as well as the theoretic and methodological differences (e.g., heterogeneity in measures) complicate comparisons. Conflicting results may be at least partially due to differences in methods and underlying assumptions.

\section{REFERENCES}

[1] Ursin H, Eriksen HR. The cognitive activation theory of stress. Psychoneuroendocrinology 2004; 29: 567-592.

[2] Mason JW. A historical view of the stress field part 2. J Human Stress 1975; 1: 22-36 concl.

[3] Mason JW. A historical view of the stress field part 1. J Human Stress 1975; 1: 6-12 contd.

[4] Saper CB, Scammell TE, Lu J. Hypothalamic regulation of sleep and circadian rhythms. Nature 2005; 437: 1257-1263.

[5] Chrousos GP. Stress and disorders of the stress system. Nat Rev Endocrinol 2009; 5: 374-381.

[6] Van Cauter E. temp- in sleep chapter. Presented in draft 100621 from Anne Helene. 2005.

[7] Weitzman ED, Zimmerman JC, Czeisler CA, Ronda J. Cortisol secretion is inhibited during sleep in normal man. J Clin Endocrinol Metab 1983; 56: 352-358.

[8] Steiger A. Sleep and the hypothalamo-pituitary-adrenocortical system. Sleep Med Rev 2002; 6: 125-138.

[9] Steiger A, Antonijevic IA, Bohlhalter S, Frieboes RM, Friess E, Murck H. Effects of hormones on sleep. Horm Res 1998; 49: 125-130.

[10] Wust S, Wolf J, Hellhammer DH, Federenko I, Schommer N, Kirschbaum C. The cortisol awakening response normal values and confounds. Noise Health 2000; 2: 79-88.

[11] Backhaus J, Junghanns K, Hohagen F. Sleep disturbances are correlated with decreased morning awakening salivary cortisol. Psychoneuroendocrinology 2004; 29: 1184-1191.

[12] Ekstedt M, Akerstedt T, Soderstrom M. Microarousals during sleep are associated with increased levels of lipids, cortisol, and blood pressure. Psychosom Med 2004; 66: 925-931.

[13] Federenko I, Wust S, Hellhammer DH, Dechoux R, Kumsta R, Kirschbaum C. Free cortisol awakening responses are influenced by awakening time. Psychoneuroendocrinology 2004; 29: 174-184.

[14] Schlotz W, Hellhammer J, Schulz P, Stone AA. Perceived work overload and chronic worrying predict weekend-weekday differences in the cortisol awakening response. Psychosom Med 2004; 66: 207-214.

[15] Wright CE, Erblich J, Valdimarsdottir HB, Bovbjerg DH. Poor sleep the night before an experimental stressor predicts reduced NK cell mobilization and slowed recovery in healthy women. Brain Behav Immun 2007; 21: 358-363.

[16] Griefahn B, Robens S. The cortisol awakening response: a pilot study on the effects of shift work, morningness and sleep duration. Psychoneuroendocrinology 2008; 33: 981-988.

[17] Gustafsson K, Lindfors P, Aronsson G, Lundberg U. Relationships between self-rating of recovery from work and morning salivary cortisol. J Occup Health 2008; 50: 24-30. 
[18] Liberzon J, Abelson JL, King A, Liberzon I. Naturalistic stress and cortisol response to awakening: adaptation to seafaring. Psychoneuroendocrinology 2008; 33: 1023-1026.

[19] Hsiao FH, Yang TT, Ho RT, Jow GM, Ng SM, Chan CL, et al. The self-perceived symptom distress and healthrelated conditions associated with morning to evening diurnal cortisol patterns in outpatients with major depressive disorder. Psychoneuroendocrinology 2009; 35: 503-515.

[20] Kumari M, Badrick E, Ferrie J, Perski A, Marmot M, Chandola T. Self-reported sleep duration and sleep disturbance are independently associated with cortisol secretion in the Whitehall II study. J Clin Endocrinol Metab 2009; 94: 4801-4809.

[21] Stalder T, Evans P, Hucklebridge F, Clow A. Associations between psychosocial state variables and the cortisol awakening response in a single case study. Psychoneuroendocrinology 2010; 35: 209-214.

[22] Vreeburg SA, Kruijtzer BP, van Pelt J, van Dyck R, DeRijk RH, Hoogendijk WJ, et al. Associations between sociodemographic, sampling and health factors and various salivary cortisol indicators in a large sample without psychopathology. Psychoneuroendocrinology 2009; 34: 1109-1120.

[23] Bailey SL, Heitkemper MM. Morningness-eveningness and early-morning salivary cortisol levels. Biol Psychol 1991; 32: 181-192.

[24] Dahlgren A, Kecklund G, Theorell T, Akerstedt T. Day-to-day variation in saliva cortisol-relation with sleep, stress and self-rated health. Biol Psychol 2009; 82: 149-155.

[25] Lasikiewicz N, Hendrickx H, Talbot D, Dye L. Exploration of basal diurnal salivary cortisol profiles in middleaged adults: associations with sleep quality and metabolic parameters. Psychoneuroendocrinology 2008; 33: 143-151.

[26] Dettenborn L, Rosenloecher F, Kirschbaum C. No effects of repeated forced wakings during three consecutive nights on morning cortisol awakening responses (CAR): a preliminary study. Psychoneuroendocrinology 2007; 32: 915-921.

[27] Spiegel K, Leproult R, Van Cauter E. Impact of sleep debt on metabolic and endocrine function. Lancet 1999; 354: 1435-1439.

[28] Heiser P, Dickhaus B, Schreiber W, Clement HW, Hasse C, Hennig J, et al. White blood cells and cortisol after sleep deprivation and recovery sleep in humans. Eur Arch Psychiatry Clin Neurosci 2000; 250: 16-23.

[29] Goh VH, Tong TY, Lim CL, Low EC, Lee LK. Effects of one night of sleep deprivation on hormone profiles and performance efficiency. Mil Med 2001; 166: 427-431.

[30] Pagani M, Pizzinelli P, Traon AP, Ferreri C, Beltrami S, Bareille MP, et al. Hemodynamic, autonomic and baroreflex changes after one night sleep deprivation in healthy volunteers. Auton Neurosci 2009; 145: 76-80.

[31] van Leeuwen WM, Lehto M, Karisola P, Lindholm H, Luukkonen R, Sallinen M, et al. Sleep restriction increases the risk of developing cardiovascular diseases by augmenting proinflammatory responses through IL17 and CRP. PLoS One 2009; 4: e4589.

[32] Birchler-Pedross A, Schroder CM, Munch M, Knoblauch V, Blatter K, Schnitzler-Sack C, et al. Subjective wellbeing is modulated by circadian phase, sleep pressure, age, and gender. J Biol Rhythms 2009; 24: 232-242.

[33] Harvey AG, Tang NK, Browning L. Cognitive approaches to insomnia. Clin Psychol Rev 2005; 25: 593-611. 